\title{
Laparoscopic duodenal segmental resection and duodenojejunostomy for symptomatic duodenal diverticula in three cases treated at a community hospital
}

\author{
Yong Jun Jeon, Eui Chul Jeong \\ Department of Surgery, Chinjujeil Hospital, Jinju, Korea
}

\begin{abstract}
The duodenum is the second most common site of diverticula following the colon, but is associated with fewer complications than colonic diverticula. Diverticulitis, cholangitis, pancreatitis, perforation, hemorrhage, and blind loop syndrome may occur as complications of duodenal diverticula. Although nonoperative treatment is an option for patients in good condition without signs of sepsis, surgery is generally required for definitive treatment of complications. There are several surgical procedures for symptomatic duodenal diverticula. We performed laparoscopic duodenal segmental resection and duodenojejunostomy without open conversion in three cases. We believe that this procedure is ideal for cases of symptomatic duodenal diverticula when performed by an experienced surgeon with the goal of definitive treatment.
\end{abstract}

Keywords: Duodenum, Diverticulum, Diverticulitis, Laparoscopy, Duodenojejunostomy
Received March 4, 2020

Revised August 4, 2020

Accepted October 14, 2020

Corresponding author

Eui Chul Jeong

Department of Surgery, Chinjujeil

Hospital, 885 Jinju-daero, Jinju 52709,

Korea

Tel: +82-55-750-7123

Fax: +82-55-750-7574

E-mail:surgchul@empas.com ORCID:

https://orcid.org/0000-0002-4361-4745

Copyright (C) 2021 The Journal of Minimally Invasive Surgery. All rights reserved.

This is an Open Access article distributed under the terms of the Creative Commons Attribution Non-Commercial License (http:// creativecommons.org/licenses/by-nc/4.0/) which permits unrestricted non-commercial use, distribution, and reproduction in any medium, provided the original work is properly cited.

\section{INTRODUCTION}

Upper gastrointestinal case-series have reported duodenal diverticula (DD) in $1 \%$ to $5 \%$ of cases, whereas some autopsy series have reported an incidence as high as $22 \%[1,2]$. Most cases of DD are asymptomatic and benign, and less than $5 \%$ of cases of DD require surgery [1]. Depending on the patient's condition, the attending doctor may recommend surgical or nonsurgical treatment for symptomatic DD. The surgical procedure used to treat symptomatic DD may depend on its anatomical complexity. However, surgery may increase the risk of morbidity and mortality due to surgical complications that may include bile duct injury, pancreatitis, duodenal leak, fistula, abscess, and persistent sepsis [2]. Minimally invasive surgical procedures are becoming increasingly popular. Here, we report three cases of patients with symptomatic DD who underwent successful laparoscopic duodenal segmental resection (LDSR) and duodenojejunostomy (DJ) without open conversion.

\section{CASE REPORT}

\section{Case 1}

A 56-year-old female was transferred from another hospital due to epigastric pain persisting for 17 days. She had no history of an underlying disease or prior surgery. The initial laboratory examination revealed that C-reactive protein (CRP) was 44.7 $\mathrm{mg} / \mathrm{L}$ (reference value, $0.0-10.0 \mathrm{mg} / \mathrm{L}$ ), but other laboratory tests were unremarkable. The initial computed tomography (CT) scan revealed a $4 \mathrm{~cm}$-sized extraluminal outpouching in the third/ 
fourth portion of the duodenum with infiltration that was suspected of being duodenal diverticulitis or inflamed duplication cyst. LDSR and DJ was performed 3 days after admission. The detailed surgical procedure is below.

(1) A total of five trocars were inserted. A 12-mm ballooning trocar was inserted at the infra-umbilical area. Two 5-mm trocars were inserted at the right upper quadrant for an assistant. A 5-mm trocar and another 12-mm ballooning trocar were inserted at the left upper quadrant for an operator (Fig. 1).

(2) Open the parietal peritoneum of mesocolon from the right side of the Treitz ligament to the right side of the duodenal $\mathrm{C}$ loop, and expose the second and third portion of the duodenum.

(3) Open the parietal peritoneum at the Treitz ligament completely, and mobilize the duodenum from its distal portion.

(4) Divide the mesenteric vessels from the superior mesenteric

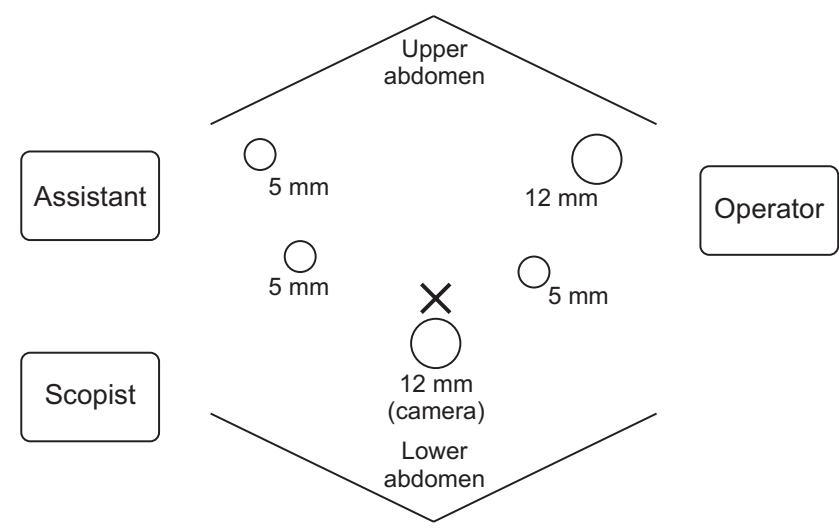

Fig. 1. A 12-mm ballooning trocar was inserted at the infra-umbilical area for a scopist. Two 5-mm trocars were inserted at the right upper quadrant of the abdomen for an assistant. A 5-mm trocar and another 12-mm ballooning trocar were inserted at the left upper quadrant of the abdomen for an operator. The "X" mark indicates the umbilicus.
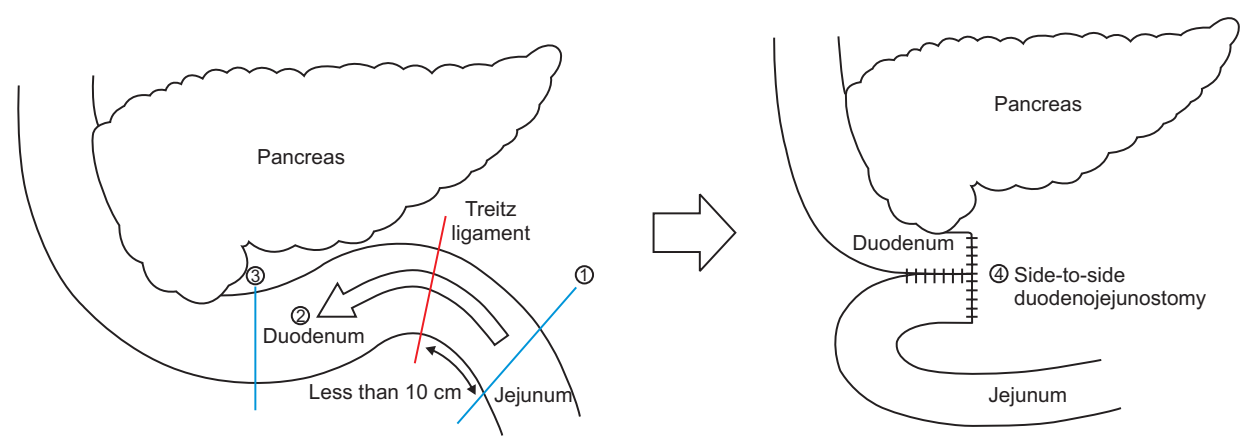

Fig. 2. (1) The proximal jejunum was transected by a laparoscopic stapler (Endo-GIA 45 mm; Medtronic, Minneapolis, MN, USA). (2) From the transected jejunum, the duodenum and the jejunum were dissected to the second/third portion of the duodenum. (3) The second/third portion of the duodenum was transected by a laparoscopic stapler (Endo-GIA $45 \mathrm{~mm}$ ). (4) Duodenojejunostomy was performed with side-to-side pattern by two laparoscopic staplers (Endo-GIA $45 \mathrm{~mm}$ ) and laparoscopic hand-sewn reinforcement.
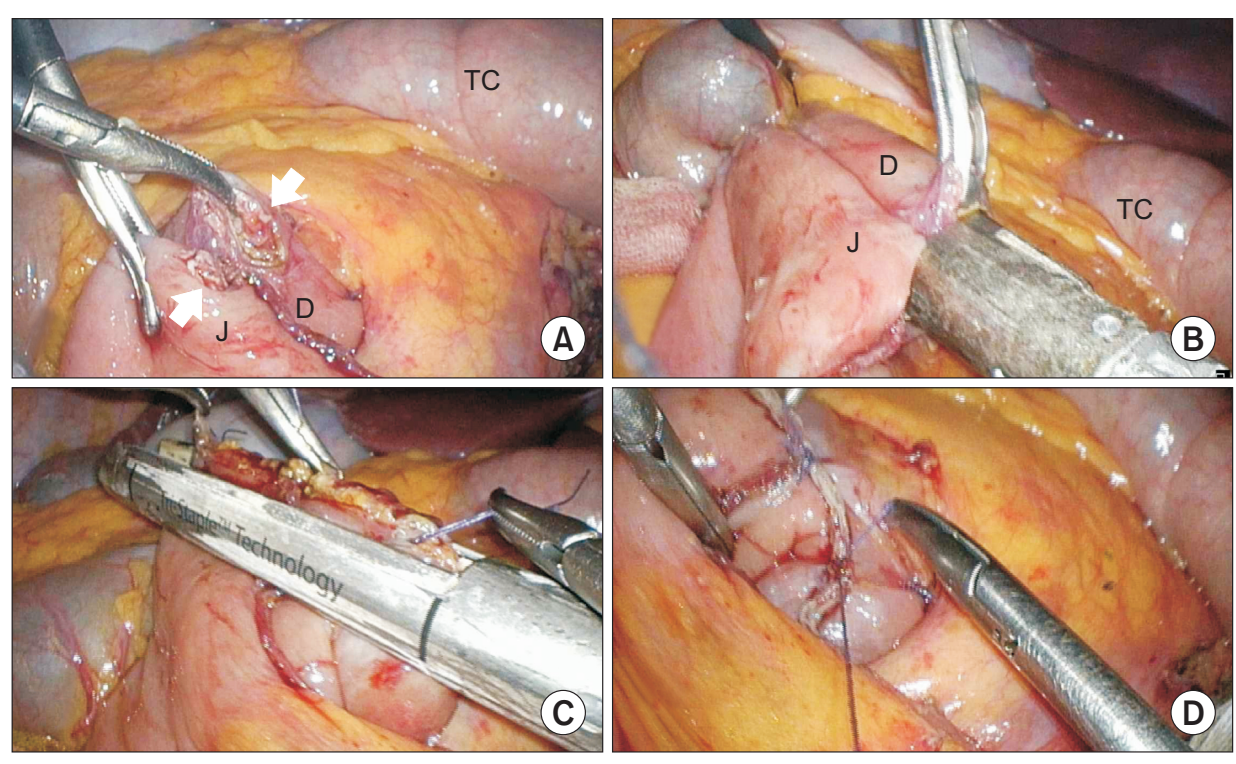

Fig. 3. (A) Entry holes (arrows) were made. (B) Side-to-side anastomosis was performed by a laparoscopic stapler (Endo-GIA 45 mm; Medtronic, Minneapolis, MN, USA). (C) The entry holes were closed by another laparoscopic stapler (Endo-GIA 45 mm). (D) The stapler lines were reinforced by laparoscopic handsewn method. D, duodenum; J, jejunum; $\mathrm{TC}$, transverse colon. 
artery and vein along with the duodenum, and mobilize the distal third portion of the duodenum.

(5) Transect the proximal jejunum with a 45-mm laparoscopic stapler (Fig. 2).

(6) Move the proximal jejunum and the distal duodenum to right side through the posterior side of superior mesenteric artery and vein.

(7) Separate the duodenum containing the duodenal diverticula from the surroundings to the right side.

(8) Transect the proximal duodenum including the diverticulum with a 45-mm laparoscopic stapler.
(9) Performed side-to-side anastomosis of the duodenum and the jejunum with a 45-mm laparoscopic stapler (Fig. 3).

(10) Close the staple opening site with delta shape anastomosis by another 45-mm laparoscopic stapler.

The surgery confirmed that the diverticulum was located in the fourth portion of the duodenum and inflammation extended to the superior mesenteric vessels (Fig. 4A). She was discharged on postoperative day (POD) 10 without complications (Table 1). The pathologic diagnosis was a diverticulum with perforation and abscess. She has not experienced any prominent abdominal symptoms 4 years after the surgery.
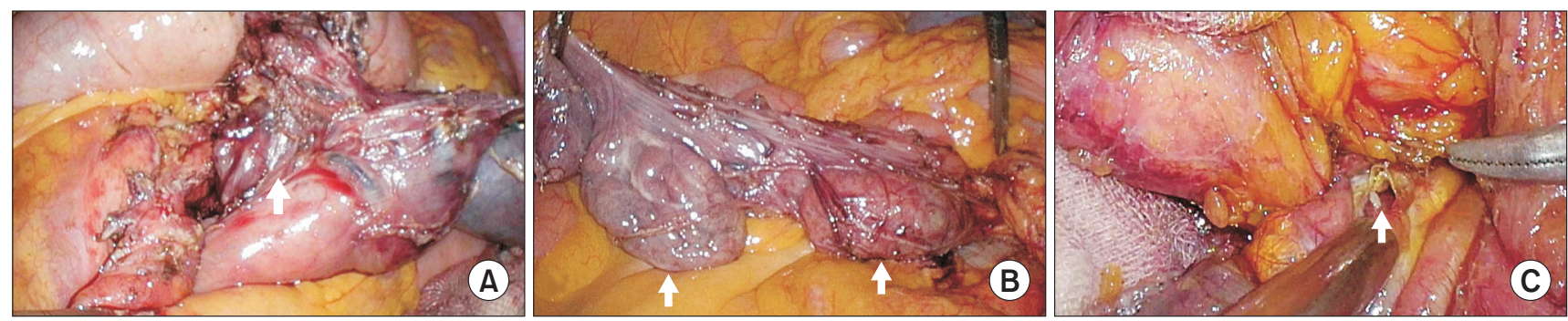

Fig. 4. (A) Case 1: the intraoperative picture shows a diverticulum (arrow) in the duodenum. The proximal jejunum was already transected. (B) Case 2: the intraoperative picture shows two diverticula (arrow) in the duodenum. The proximal jejunum was already transected. (C) Case 3: the intraoperative picture shows perforation of the diverticulum (arrow) in the duodenum. The second portion of the duodenum and the proximal jejunum were already transected.

Table 1. Preoperative characteristics, intraoperative details and postoperative course of the patients

\begin{tabular}{|c|c|c|c|}
\hline Variable & Case 1 & Case 2 & Case 3 \\
\hline Age (yr) & 56 & 76 & 70 \\
\hline Sex & Female & Female & Male \\
\hline Body mass index $\left(\mathrm{kg} / \mathrm{m}^{2}\right)$ & 21.49 & 21.5 & 27.48 \\
\hline Underlying disease & None & None & Hypertension, dyslipidemia, BPH \\
\hline Operation history & None & Laparoscopic rectopexy due to rectal prolapse & None \\
\hline ASA PS classification & $\|$ & $\|$ & $\|$ \\
\hline Location of duodenal diverticula & 4th portion & 2nd and 3rd portion & 3rd portion \\
\hline \multirow[t]{3}{*}{ Name of operation } & LDSR and DJ & LDSR and DJ & LDSR and DJ \\
\hline & & Diverticulectomy & Cholecystectomy \\
\hline & & & Marsupialization of the right renal cyst \\
\hline Operation time $(\mathrm{min})^{\mathrm{a})}$ & 225 & 220 & 452 \\
\hline Open conversion & None & None & None \\
\hline Postoperative stay (day) & 10 & 21 & 48 \\
\hline Hospital stay (day) & 13 & 22 & 51 \\
\hline Complication & None & None & Abscess, delirium \\
\hline
\end{tabular}

BPH, benign prostate hyperplasia; ASA PS, American Society of Anesthesiologists physical status; LDSR, laparoscopic duodenal segmental resection; DJ, duodenojejunostomy.

${ }^{\text {a)}}$ Operation time was calculated as the time from first incision to final skin closure. 


\section{Case 2}

A 76-year-old female visited our outpatient department due to dyspepsia and epigastric discomfort persisting for 5 months. Her vital signs, including blood pressure, heart rate, respiratory rate, and body temperature, were stable. Her initial laboratory tests were unremarkable. A CT scan performed 5 years earlier revealed large duodenal diverticula in the third and fourth portions and a preoperative CT scan showed no definite interval change in the diverticula compared to previous study. She underwent surgery 1 day after admission. LDSR and DJ was performed for the larger diverticulum with similar pattern like case 1 and diverticulectomy was performed for the smaller diverticulum. The surgery revealed that the larger diverticulum was located in the third portion and a smaller diverticulum was located in the distal second portion (Fig. 4B). She was discharged on POD 21 without complications (Table 1). The pathologic diagnosis was two diverticula, but did not mention diverticulitis. She has not experienced any prominent abdominal symptoms 9 months after the surgery.

\section{Case 3}

A 70-year-old male visited our emergency department due to abdominal pain lasting 7 days. His systolic blood pressure and diastolic blood pressure were 105 and $66 \mathrm{mmHg}$, respectively. His respiratory rate was 20 breaths/min. However, his heart rate was 122 beats/min and his body temperature was $37.7^{\circ} \mathrm{C}$. His white blood cell count was $22,340 / \mu \mathrm{L}$ (reference value, 4,000-10,000/ $\mu \mathrm{L}$ ), CRP level was $346.2 \mathrm{mg} / \mathrm{L}$, potassium level was $3.3 \mathrm{mmol} /$ $\mathrm{L}$ (reference value, $3.5-5.1 \mathrm{mmol} / \mathrm{L}$ ), and blood urea nitrogen was $29.9 \mathrm{mg} / \mathrm{dL}$ (reference value, $6-23 \mathrm{mg} / \mathrm{dL}$ ) at the initial laboratory examination. His concomitant disorders were hypertension, dyslipidemia, and benign prostate hyperplasia, and prior tuberculosis had been cured. An initial CT scan revealed a duodenal diverticulum in the third portion with free air and infiltration, suspected of being a duodenal diverticulum perforation. He was initially treated with bowel rest, administration of intravenous fluid, parenteral nutrition and antibiotic. However, 3 days after admission, we decided to perform surgery because he had developed signs of shock and follow-up CT scan revealed more aggravated state of the right pararenal space inflammation and fluid extension. The surgical procedure was performed similar pattern with case 1 . Meanwhile, the right renal cyst was included in the surgical field. LDSR and DJ with cholecystectomy and marsupialization of the right renal cyst were performed. The surgery revealed that the perforation was located in the third portion of the duodenum (Fig. 4C), and that inflammation and purulent fluid had extended to the gallbladder and retroperitoneal space. Percutaneous drainage was performed on POD 4 and 11 due to an abscess in the right pararenal space. He suffered from delirium, and was newly diagnosed with hyperthyroidism and benign paroxysmal positional vertigo during hospitalization. He was discharged on POD 48 (Table 1). The pathologic diagnosis was a diverticulum with perforation. He has not experienced any prominent abdominal symptom 8 months after the surgery.

\section{DISCUSSION}

A diverticulum is an outpouching of a hollow structure and can be classified as congenital or acquired, intraluminal or extraluminal, and true or false depending on the layer involved [1]. True diverticula contain all layers of the structure, whereas false diverticula do not involve the muscular layers or adventitia [1]. False diverticula, in the gastrointestinal tract for example, only involve the submucosa and mucosa. The duodenum is the second most common site of diverticula following the colon [1]. Twothirds to three-quarters of diverticula are found within a $2-\mathrm{cm}$ radius of the ampulla and project from the medial duodenal wall [1].

DD are twice as common in women as in men, and is rare under 40 years of age [1]. Symptoms occur in $5 \%$ to $10 \%$ of patients with DD [3]. The clinical presentation varies significantly and it requires a high degree of suspicion. Major complications of DD include inflammation; obstruction of the biliary or pancreatic duct that may contribute to cholangitis or pancreatitis, respectively; perforation; hemorrhage; and rarely, blind loop syndrome $[1,3]$. Compared with colonic diverticula, DD are generally a less common cause of inflammation because of its larger size, faster intraluminal flow, and lower bacterial count [4]. Although perforation is rare, it is a life-threatening complication associated with a mortality rate of up to $30 \%$ [5]. Perforation was found in two of the three cases described in our report. The most common cause of perforation is diverticulitis [3]. Symptoms of a perforated diverticulum are ambiguous and nonspecific, and can be confused with other intra-abdominal diseases, such as peptic ulcer disease, pancreatitis, cholecystitis, colitis, or retrocecal appendicitis [4] The most challenging differential diagnosis is a perforated duodenal ulcer. However, these can be differentiated by the site of perforation because most ulcers involve the duodenal bulb or the first portion whereas diverticula are usually located in the second or third portion [6].

Nonoperative treatment of perforated diverticula was first reported in 1963 [7], and it may be appropriate for patients in good condition without signs of sepsis [2]. However, several surgical procedures are available for symptomatic DD $[1,3]$ and surgery is generally the standard treatment for perforated DD [8]. Stapled or hand-sewn diverticulectomy with drainage of the retroperitoneal space is the most common procedure to repair the lesion [3]. Isolated drainage of retroperitoneum, laparoscopic 
diverticulectomy, reinforcement with an omental patch, diversion of enteric flow with gastrojejunostomy or DJ, pancreaticoduodenectomy have also been described $[1,3,8]$. In our opinion, the benefit of LDSR and DJ is that surgeons can perform definite treatment without possibility of recurrence as it removes a diseased segment completely. However, LDSR and DJ takes longer time than simple drainage procedure or diverticulectomy due to anatomical complexity, surgeons should have enough knowledge and experience about the anatomy and the procedure. Important thing performing in LDSR and DJ is that dissection should begin from less- or noninflamed tissue, such as jejunum, to find out exact surgical plane. To our knowledge, this is first case-series to describe the use of LDSR and DJ to treat symptomatic DD.

In conclusion, LDSR and DJ is a potential surgical treatment option for patients with symptomatic DD, when performed by an experienced surgeon with the goal of definitive treatment.

\section{NOTES}

\section{Ethical statements}

We conducted this study in compliance with the principles of the Declaration of Helsinki. Research on this case-series was approved by Korea National Institute for Bioethics Policy (approval number: P01-201909-21-012). Because all data were retrospectively collected from clinical records and imaging systems, the need for informed consent was waived by the ethics committee.

\section{Authors' contributions}

Conceptualization: ECJ

Formal analysis: YJJ

Methodology: YJJ

Writing-original draft: YJJ

Writing-review \& editing: All authors
All authors read and approved the final manuscript.

\section{Conflict of interest}

All authors have no conflicts of interest to declare.

\section{ORCID}

Yong Jun Jeon, https://orcid.org/0000-0001-9023-8394

Eui Chul Jeong, https://orcid.org/0000-0002-4361-4745

\section{REFERENCES}

1. Townsend CM, Sabiston DC. Sabiston textbook of surgery : the biological basis of modern surgical practice. 19th ed. Philadelphia (PA): Elsevier Saunders; 2012.

2. Kim KH, Park SH. Conservative treatment of duodenal diverticulitis perforation: a case report and literature review. Open Access Emerg Med 2018;10:101-104.

3. Thorson CM, Paz Ruiz PS, Roeder RA, Sleeman D, Casillas VJ. The perforated duodenal diverticulum. Arch Surg 2012;147:81-88.

4. Coulier B, Maldague P, Bourgeois A, Broze B. Diverticulitis of the small bowel: CT diagnosis. Abdom Imaging 2007;32:228-233.

5. Bergman S, Koumanis J, Stein LA, Barkun JS, Paraskevas S. Duodenal diverticulum with retroperitoneal perforation. Can J Surg 2005;48: 332.

6. Ames JT, Federle MP, Pealer KM. Perforated duodenal diverticulum: clinical and imaging findings in eight patients. Abdom Imaging 2009; 34:135-139.

7. Shackleton ME. Perforation of a duodenal diverticulum with massive retroperitoneal emphysema. N Z Med J 1963;62:93-94.

8. Costa Simões V, Santos B, Magalhães S, Faria G, Sousa Silva D, Davide J. Perforated duodenal diverticulum: Surgical treatment and literature review. Int J Surg Case Rep 2014;5:547-550. 